\title{
LIMSUP RESULTS AND A GENERALIZED UNIFORM LIL FOR AN LPQD SEQUENCE
}

\author{
Yong-Kab Choi, Kyo-Shin Hwang, And Hee-Jin Moon
}

\begin{abstract}
In this paper we establish some limsup results and a generalized uniform law of the iterated logarithm (LIL) for the increments of partial sums of a strictly stationary and linearly positive quadrant dependent (LPQD) sequence of random variables.
\end{abstract}

\section{Introduction and results}

In the last years there has been growing interest in concepts of positive/negative dependence for random sequences. Lehmann [5] introduced a definition of positive dependence:

Definition 1.1. Two random variables $X$ and $Y$ are said to be positive quadrant dependent (PQD, for short) if

$$
P(X>x, Y>y) \geq P(X>x) P(Y>y) \quad \text { for all } x, y \in \mathbb{R} .
$$

A much stronger concept than PQD was considered by Esary, Proschan and Walkup [4]:

Definition 1.2. A finite family $\left\{\xi_{1}, \ldots, \xi_{n}\right\}$ of random variables is said to be positively associated (PA, for short) if for every pair of subsets $A$ and $B$ of $\{1,2, \ldots, n\}$,

$$
\operatorname{Cov}\left(f\left(\xi_{i} ; i \in A\right), g\left(\xi_{j} ; j \in B\right)\right) \geq 0
$$

whenever $f$ and $g$ are coordinatewise nondecreasing and the covariance exists. An infinite family is PA if every finite subfamily is PA.

Another notion of positive dependence which is stronger than PQD and weaker than PA, is due to Newman [7]:

Received September 20, 2010; Revised August 1, 2011.

2010 Mathematics Subject Classification. 60F15, 60G17.

Key words and phrases. linearly positive quadrant dependence, strictly stationary sequence, law of the iterated logarithm.

The first author was supported by NRF-2011-0025948.

The third author was supported by NRF-2009-353-C00012. 
Definition 1.3. A sequence $\left\{\xi_{j} ; j \geq 1\right\}$ of random variables is said to be linearly positive quadrant dependent (LPQD, for short) if for any disjoint finite subsets $A, B \subset\{1,2, \ldots\}$ and any positive $r_{j}$ 's, $\sum_{i \in A} r_{i} \xi_{i}$ and $\sum_{j \in B} r_{j} \xi_{j}$ are PQD.

Recently, Li and Wang [6] obtained the following law of the iterated logarithm for an LPQD random sequence (cf. Theorem A below).

Let $\left\{\xi_{j}, j \geq 1\right\}$ be a strictly stationary LPQD sequence with $E \xi_{1}=0$ and $E \xi_{1}^{2}>0$, which satisfies conditions

(i) $E\left|\xi_{1}\right|^{p}<\infty$ for $p>2$,

(ii) $u(n):=\sum_{j=n+1}^{\infty} \operatorname{Cov}\left(\xi_{1}, \xi_{j}\right)=O\left(n^{-\lambda}\right)$ for some $\lambda>2$, where $n \geq 1$,

(iii) $\sigma^{2}:=E \xi_{1}^{2}+2 \sum_{j=2}^{\infty} \operatorname{Cov}\left(\xi_{1}, \xi_{j}\right)<\infty$.

Define a sum and the increments of partial sums of the sequence $\left\{\xi_{j}\right\}$, respectively, as follows:

$$
S(x)=\sum_{j=1}^{[x]} \xi_{j}, \quad S(0)=0 \quad \text { and } \quad S_{u}(t)=\sum_{k=[u]+1}^{[u+t]} \xi_{k}, \quad u \geq 0, t \geq 1,
$$

where $[x]$ denotes the integer part of $x \geq 1$. Observe that $S_{0}(x)=S(x)$.

Theorem A. Let $\left\{\xi_{j}, j \geq 1\right\}$ be a strictly stationary LPQD sequence with $E \xi_{1}=0$ and $E \xi_{1}^{2}>0$, which satisfies conditions (i)-(iii) above. Then

$$
\limsup _{n \rightarrow \infty} \frac{S(n)}{\sqrt{2 \sigma^{2} n \log \log n}}=1 \quad \text { a.s. }
$$

In this paper, we establish some limsup results and a generalized uniform law of the iterated logarithm (LIL) for the increments of the partial sums of a strictly stationary and linearly positive quadrant dependent (LPQD) sequence of random variables, which extend and generalize the inspiring result Theorem A due to Li and Wang [6]. On the other hand, we consider similar results as in Choi [1] for linearly negative quadrant dependent (LNQD) random sequences under another conditions.

Throughout the paper, let $\left\{\xi_{j}, j \geq 1\right\}$ be a strictly stationary LPQD sequence with $E \xi_{1}=0$ and $E \xi_{1}^{2}>0$, which satisfies conditions (i)-(iii) above. Assume that

$$
\sigma(t):=\sqrt{E S_{u}^{2}(t)}>0, \quad{ }^{\forall} u \geq 0,
$$

is a nondecreasing continuous and regularly varying function of $t \geq 1$ with exponent $\alpha$ at $\infty$ for some $0<\alpha<1$.

A positive function $R(t)$ of $t>0$ is said to be regularly varying with exponent $\alpha>0$ at $b \geq 0$ if $\lim _{t \rightarrow b}\{R(x t) / R(t)\}=x^{\alpha}$ for $x>0$.

Recall that a function $L(t)$ of $t>0$ is said to be slowly varying at $b \geq 0$ if $\lim _{t \rightarrow b}\{L(x t) / L(t)\}=1$ for $x>0$. Thus we can write the regularly varying function $R(t)$ as $R(t)=t^{\alpha} L(t)$. 
Let $\left\{\xi_{j}, j \geq 1\right\}$ be a strictly stationary LPQD sequence with $E \xi_{1}=0$, $E \xi_{1}^{2}>0$ and condition (iii). Yang and Wang [8] proved

$$
\lim _{n \rightarrow \infty} \frac{\sigma^{2}(n)}{n}=\sigma^{2}
$$

By this relation (1.2), we ensure that $\sigma(n) \approx \sigma \sqrt{n}$ for $n$ large enough. Hence $\sigma(\cdot)$ is regarded as a regularly varying function with exponent $\alpha=1 / 2$ at $\infty$. Furthermore, (1.1) can be written as

$$
\limsup _{n \rightarrow \infty} \frac{S(n)}{\sigma(n) \sqrt{2 \log \log n}}=1 \quad \text { a.s. }
$$

under the conditions (i)-(iii).

Suppose that $\left\{a_{n}, n \geq 1\right\}$ and $\left\{b_{n}, n \geq 1\right\}$ are positive nondecreasing sequences such that $a_{n} \leq b_{n}$ and $b_{n} \rightarrow \infty$ as $n \rightarrow \infty$. Denote

$$
\beta(n)=\sqrt{2\left\{\log \left(b_{n} / a_{n}\right)+\log \log b_{n}\right\}},
$$

where $\log x:=\ln (\max \{x, 1\})$. The main results are as follows:

Theorem 1.1. Let $\left\{\xi_{j}, j \geq 1\right\}$ be a strictly stationary LPQD sequence with $E \xi_{1}=0$ and $E \xi_{1}^{2}>0$, which satisfies conditions (i)-(iii) above. Assume that $\sigma(\cdot)$ is a nondecreasing continuous and regularly varying function with exponent $\alpha$ at $\infty$ for some $0<\alpha<1$. Then

$$
\begin{gathered}
\limsup _{n \rightarrow \infty} \sup _{0 \leq i \leq b_{n}} \sup _{1 \leq j \leq a_{n}} \frac{\left|S_{i}(j)\right|}{\sigma\left(a_{n}\right) \beta(n)} \leq 1 \quad \text { a.s., } \\
\quad \limsup _{n \rightarrow \infty} \frac{\left|S\left(a_{n}\right)\right|}{\sigma\left(a_{n}\right) \sqrt{2 \log \log a_{n}}} \leq 1 \quad \text { a.s. }
\end{gathered}
$$

The result (1.5) is immediate from (1.4) when $i=0$ and $j=a_{n}=b_{n}$.

Theorem 1.2. Let $\left\{\xi_{j}\right\}$ and $\sigma(\cdot)$ be as in Theorem 1.1, and let $a_{n} \leq n$. Then

$$
\limsup _{n \rightarrow \infty} \frac{S\left(a_{n}\right)}{\sigma\left(a_{n}\right) \sqrt{2 \log \log a_{n}}} \geq 1 \quad \text { a.s. }
$$

From Theorems 1.1 and 1.2 with $a_{n}=b_{n}$, we have the following limsup result:

Corollary 1.1. Under the assumptions of Theorem 1.2, we have

$$
\begin{gathered}
\limsup _{n \rightarrow \infty} \sup _{0 \leq i \leq a_{n}} \sup _{1 \leq j \leq a_{n}} \frac{\left|S_{i}(j)\right|}{\sigma\left(a_{n}\right) \sqrt{2 \log \log a_{n}}}=1 \quad \text { a.s., } \\
\quad \limsup _{n \rightarrow \infty} \frac{S\left(a_{n}\right)}{\sigma\left(a_{n}\right) \sqrt{2 \log \log a_{n}}}=1 \quad \text { a.s. }
\end{gathered}
$$

Taking $a_{n}=n$ in Corollary 1.1 yields an explicit form of the generalized uniform LIL for the increments of the partial sums of an LPQD sequence as follows. 
Corollary 1.2 (LIL). Under the assumptions of Theorem 1.2, we have

$$
\begin{gathered}
\limsup _{n \rightarrow \infty} \sup _{0 \leq i \leq n} \sup _{1 \leq j \leq n} \frac{\left|S_{i}(j)\right|}{\sigma(n) \sqrt{2 \log \log n}}=1 \quad \text { a.s., } \\
\quad \limsup _{n \rightarrow \infty} \frac{S(n)}{\sigma(n) \sqrt{2 \log \log n}}=1 \quad \text { a.s. }
\end{gathered}
$$

It is clear that the result (1.10) gives (1.1) as well as (1.3) for a strictly stationary LPQD sequence by the viewpoint of (1.2).

\section{Proofs}

In this section, let $c$ denote a positive constant which may take different values whenever it appears in different lines. We need the following properties:

$\left(P_{1}\right)$ Two random variables $X$ and $Y$ are PQD if and only if Cov $(f(X), g(Y))$ $\geq 0$ for all real nondecreasing functions $f$ and $g$ (such that $f(X)$ and $g(Y)$ have finite variances) (see Lehmann [5]);

$\left(P_{2}\right)$ (Hoeffding equality): For any absolutely continuous functions $f$ and $g$ on the real line and for any random variables $X$ and $Y$ satisfying $E f^{2}(X)+$ $E g^{2}(Y)<\infty$, we have

$$
\begin{array}{r}
\operatorname{Cov}(f(X), g(Y))=\int_{-\infty}^{\infty} \int_{-\infty}^{\infty} f^{\prime}(x) g^{\prime}(y)\{P(X \geq x, Y \geq y) \\
-P(X \geq x) P(Y \geq y)\} d x d y
\end{array}
$$

By the previous relation $\sigma(n) \approx \sigma \sqrt{n}$ for $n$ large enough, the conclusion of Lemma 2 in Li and Wang [6] can be expressed as follows:

$$
\sup _{x}\left|P\left\{\frac{S(n)}{\sigma(n)} \leq x\right\}-\Phi(x)\right|=O\left(n^{-1 / 5}\right), \quad n \rightarrow \infty
$$

where $\Phi(x)=\frac{1}{\sqrt{2 \pi}} \int_{-\infty}^{x} e^{-w^{2} / 2} d w$. Consequently, we have an analogue of Lemma 3 in the just mentioned paper:

Lemma 2.1. Let $\left\{\xi_{j}\right\}$ and $\sigma(\cdot)$ be as in Theorem 1.1. Suppose that $\{g(n), n \geq$ $1\}$ and $\left\{n_{m}, m \geq 1\right\}$ are positive nondecreasing sequences such that $\sum_{m=1}^{\infty} n_{m}^{-1 / 5}$ $<\infty$. Then the following statements are equivalent:
(A) $\sum_{m=1}^{\infty} P\left\{\frac{S\left(n_{m}\right)}{\sigma\left(n_{m}\right)}>g\left(n_{m}\right)\right\}<\infty$,
(B) $\sum_{m=1}^{\infty} P\left\{\frac{\left|S\left(n_{m}\right)\right|}{\sigma\left(n_{m}\right)}>g\left(n_{m}\right)\right\}<\infty$

and

(C) $\sum_{m=1}^{\infty} \frac{1}{g\left(n_{m}\right)} \exp \left(-\frac{1}{2} g^{2}\left(n_{m}\right)\right)<\infty$. 
The following lemma is useful for proving Theorem 1.1. The proof of Lemma 2.2 below is similar to that of Lemma 2.3 in Choi and Csörgő [2].

Lemma 2.2. Let $\mathbb{D}$ be a compact subset of $\mathbb{R}^{d}, d \geq 1$, with the Euclidean norm $\|\cdot\|$, and let $\{X(\mathbf{t}) ; \mathbf{t} \in \mathbb{D}\}$ be a separable and strictly stationary centered random field indexed only by $\mathbb{D}$. Suppose that

$$
\begin{gathered}
0<\Gamma:=\sup _{\mathbf{t} \in \mathbb{D}} \sqrt{E X^{2}(\mathbf{t})}<\infty \quad \text { and } \\
0<\sqrt{E\{X(\mathbf{t})-X(\mathbf{s})\}^{2}} \leq \varphi(\|\mathbf{t}-\mathbf{s}\|) \quad \text { for } \mathbf{t} \neq \mathbf{s} \in \mathbb{D},
\end{gathered}
$$

where $\varphi(h)$ is a nondecreasing continuous function of $h>0$. Then, for any $\lambda>0$ and $K_{1}>(2 \sqrt{2}+2) \sqrt{1+2 d \log 2}$, there is a constant $c>0$ such that

$$
\begin{aligned}
& P\left\{\sup _{\mathbf{t} \in \mathbb{D}}|X(\mathbf{t})| \geq x\left(\Gamma+K_{1} \int_{0}^{\infty} \varphi\left(\sqrt{d} \lambda 2^{-y^{2}}\right) d y\right)\right\} \\
\leq & c \frac{m(\mathbb{D})}{\lambda^{d}}\left(P \left\{\frac{|X(\mathbf{t})|}{\left.\sqrt{E X^{2}(\mathbf{t})} \geq x\right\}}\right.\right. \\
& \left.+\sum_{n=1}^{\infty} 2^{d 2^{n}} P\left\{\frac{|X(\mathbf{t})|}{\sqrt{E X^{2}(\mathbf{t})}} \geq x \sqrt{1+2 d \log 2} \cdot 2^{n / 2}\right\}\right), \quad x>0,
\end{aligned}
$$

where $m(\mathbb{D})$ denotes the Lebesgue measure of $\mathbb{D}$.

By Lemma 2.2, one can estimate an upper bound of the following large deviation probability, whose proof is similar to that of Lemma 2.2 in Choi et al. [3].

Lemma 2.3. Let $\theta>1, d \geq 1$ and $m \geq l \geq 1$. Then, for any $\varepsilon>0$, there exists a positive constant $c_{\varepsilon}$ such that

$$
\begin{aligned}
& P\left\{\sup _{0 \leq i \leq \theta^{m}} \sup _{1 \leq j \leq \theta^{l}} \frac{\left|S_{i}(j)\right|}{\sigma\left(\theta^{l}\right)} \geq u\right\} \\
\leq & c_{\varepsilon} \theta^{m-l}\left(P\left\{\frac{\left|S\left(\theta^{l}\right)\right|}{\sigma\left(\theta^{l}\right)} \geq \frac{u}{1+\varepsilon}\right\}\right. \\
& \left.+\sum_{n=1}^{\infty} 2^{d 2^{n}} P\left\{\frac{\left|S\left(\theta^{l}\right)\right|}{\sigma\left(\theta^{l}\right)} \geq \frac{u}{1+\varepsilon} \sqrt{1+2 d \log 2} \cdot 2^{n / 2}\right\}\right), \quad u>1
\end{aligned}
$$

Proof of Theorem 1.1. For a fixed $\theta>1$, let

$$
\mathbb{A}_{l, m}=\left\{n: \theta^{l-1} \leq a_{n} \leq \theta^{l}, \theta^{m-1} \leq b_{n} \leq \theta^{m}\right\},
$$

where $l$ and $m$ are integers with $m>l \geq 1$. Since $(\log w) / w$ is a decreasing function of $w>1$, we get

$$
\begin{aligned}
\inf _{n \in \mathbb{A}_{l, m}} \beta(n) & \geq\left\{2 \log \left(\left(\theta^{m-1} / \theta^{l}\right) \log \theta^{m-1}\right)\right\}^{1 / 2} \\
& \geq \theta^{-1}\left\{2 \log \left(\left(\theta^{m} / \theta^{l}\right) \log \theta^{m}\right)\right\}^{1 / 2}
\end{aligned}
$$


for sufficiently large $m$. Define $\beta_{m, l}(\theta):=\sqrt{2 \log \left(\theta^{m-l} \log \theta^{m}\right)}$ so that

$$
m \theta^{m-l} \log \theta>1 \text {. }
$$

Since $b_{n} \rightarrow \infty$ as $n \rightarrow \infty$, this is equivalent to $m \rightarrow \infty$ by the definition of $\mathbb{A}_{l, m}$. It follows from the regularity of $\sigma(\cdot)$ with exponent $\alpha(0<\alpha<1)$ that

$$
\frac{\sigma\left(a_{n}\right)}{\theta^{-\alpha} \sigma\left(\theta^{l}\right)} \geq 1 \quad \text { as } n(\text { or } l) \rightarrow \infty
$$

and hence

$$
\begin{aligned}
& \limsup _{n \rightarrow \infty} \sup _{0 \leq i \leq b_{n}} \sup _{1 \leq j \leq a_{n}} \frac{\left|S_{i}(j)\right|}{\sigma\left(a_{n}\right) \beta(n)} \\
\leq & \limsup _{m \rightarrow \infty} \sup _{n \in \mathbb{A}_{l, m}} \sup _{0 \leq i \leq b_{n}} \sup _{1 \leq j \leq a_{n}} \frac{\left|S_{i}(j)\right|}{\sigma\left(a_{n}\right) \beta(n)} \\
\leq & \theta^{\alpha+1} \limsup _{m \rightarrow \infty} \sup _{0 \leq i \leq \theta^{m}} \sup _{1 \leq j \leq \theta^{l}} \frac{\left|S_{i}(j)\right|}{\sigma\left(\theta^{l}\right) \beta_{m, l}(\theta)} .
\end{aligned}
$$

Using Lemma 2.3, it follows that, for any $\varepsilon>0$,

$$
\begin{aligned}
& P\left\{\sup _{0 \leq i \leq \theta^{m}} \sup _{1 \leq j \leq \theta^{l}} \frac{\left|S_{i}(j)\right|}{\sigma\left(\theta^{l}\right) \beta_{m, l}(\theta)}>1+2 \varepsilon\right\} \\
\leq & c_{\varepsilon} \theta^{m-l}\left(P\left\{\frac{\left|S\left(\theta^{l}\right)\right|}{\sigma\left(\theta^{l}\right)}>\frac{(1+2 \varepsilon) \beta_{m, l}(\theta)}{1+\varepsilon}\right\}\right. \\
& \left.+\sum_{n=1}^{\infty} 2^{d 2^{n}} P\left\{\frac{\left|S\left(\theta^{l}\right)\right|}{\sigma\left(\theta^{l}\right)}>\frac{(1+2 \varepsilon) \beta_{m, l}(\theta)}{1+\varepsilon} \sqrt{1+2 d \log 2} \cdot 2^{n / 2}\right\}\right)
\end{aligned}
$$

for all large $m$. Now let us apply Lemma 2.1 with $n_{m}=\theta^{m}, m>1$, and

$$
g\left(\theta^{m}\right)=\frac{(1+2 \varepsilon) \beta_{m, l}(\theta)}{1+\varepsilon} \quad\left(\text { or } \quad \frac{(1+2 \varepsilon) \beta_{m, l}(\theta)}{1+\varepsilon} \sqrt{1+2 d \log 2} \cdot 2^{n / 2}\right) .
$$

Considering the right hand side of (2.4) and (C) of Lemma 2.1, we have

$$
\begin{aligned}
& \sum_{m=2}^{\infty} \frac{1+\varepsilon}{(1+2 \varepsilon) \sqrt{2 \log \left(\theta^{m-l} \log \theta^{m}\right)}} \exp \left(-\frac{1}{2}\left(\frac{1+2 \varepsilon}{1+\varepsilon}\right)^{2} 2 \log \left(\theta^{m-l} \log \theta^{m}\right)\right) \\
\leq & c \sum_{m=2}^{\infty}\left(\theta^{m-l} \log \theta^{m}\right)^{-1-\varepsilon^{\prime}}<\infty,
\end{aligned}
$$

where $\varepsilon^{\prime}=\varepsilon /(1+\varepsilon)$, and also

$$
\begin{aligned}
& \sum_{n=1}^{\infty} 2^{d 2^{n}} \sum_{m=2}^{\infty} \exp \left(-\frac{1}{2}\left(\frac{1+2 \varepsilon}{1+\varepsilon}\right)^{2} 2 \log \left(\theta^{m-l} \log \theta^{m}\right)(1+2 d \log 2) 2^{n}\right) \\
\leq & \sum_{m=2}^{\infty} \sum_{n=1}^{\infty} 2^{d 2^{n}} \exp \left(-\left(1+\varepsilon^{\prime}\right)(1+2 d \log 2) 2^{n} \log \left(\theta^{m-l} \log \theta^{m}\right)\right)
\end{aligned}
$$




$$
\begin{aligned}
& =\sum_{m=2}^{\infty} \sum_{n=1}^{\infty} 2^{d 2^{n}}\left(\theta^{m-l} \log \theta^{m}\right)^{-\left(1+\varepsilon^{\prime}\right)(1+2 d \log 2) 2^{n}} \\
& \leq c \sum_{m=2}^{\infty} \theta^{-\left(1+\varepsilon^{\prime}\right)(m-l)} \sum_{n=1}^{\infty} 2^{d 2^{n}} m^{-\left(1+\varepsilon^{\prime}\right) 2 d(\log 2) 2^{n}}(\log \theta)^{-\left(1+\varepsilon^{\prime}\right)} \\
& \leq c \sum_{m=2}^{\infty} \sum_{n=1}^{\infty} 2^{d 2^{n}-\left(\log _{2} m\right) 2 d(\log 2) 2^{n}} \\
& =c \sum_{m=2}^{\infty} \sum_{n=1}^{\infty} 2^{d 2^{n}\left(1-\left(\log _{2} m\right) \log 4\right)} \\
& \leq c \sum_{m=2}^{\infty} \sum_{n=1}^{\infty} m^{-1-\varepsilon^{\prime}} 2^{-n}<\infty
\end{aligned}
$$

It follows from (2.4) and Lemma 2.1 that

$$
\sum_{m=2}^{\infty} P\left\{\sup _{0 \leq i \leq \theta^{m}} \sup _{1 \leq j \leq \theta^{l}} \frac{\left|S_{i}(j)\right|}{\sigma\left(\theta^{l}\right) \beta_{m, l}(\theta)}>1+2 \varepsilon\right\}<\infty .
$$

Thus the Borel-Cantelli lemma yields

$$
\limsup _{m \rightarrow \infty} \sup _{0 \leq i \leq \theta^{m}} \sup _{1 \leq j \leq \theta^{l}} \frac{\left|S_{i}(j)\right|}{\sigma\left(\theta^{l}\right) \beta_{m, l}(\theta)} \leq 1+2 \varepsilon \quad \text { a.s. }
$$

This and (2.3) imply (1.4) since $\theta$ and $\varepsilon$ are arbitrary.

Proof of Theorem 1.2. Since $a_{n} \leq n$ by the assumption, it is clear that

$$
1 / \sqrt{2 \log \log a_{n}} \geq 1 / \sqrt{2 \log \log n} .
$$

Thus, for a subsequence $\left\{n_{m}, m \geq 1\right\}$ of $\{n, n \geq 1\}$, we are to verify the theorem by setting $a_{n_{m}}=n_{m}=N^{m}, N>2$. For convenience, put $a_{m}=a_{n_{m}}$. The proof of (1.6) is completed if we show that

$$
\limsup _{m \rightarrow \infty} \frac{S\left(a_{m}\right)}{\sigma\left(a_{m}\right) \sqrt{2 \log \log a_{m}}}>1-4 \varepsilon \quad \text { a.s. }
$$

for any small $\varepsilon>0$. Let

$$
B_{m}=\left\{\frac{S\left(a_{m}\right)-S\left(a_{m / 2}\right)}{\sigma\left(a_{m}-a_{m / 2}\right)}>(1-2 \varepsilon) \sqrt{2 \log \log \left(a_{m}-a_{m / 2}\right)}\right\} .
$$

In order to apply Lemma 2.1 , set $g\left(n_{m}\right)=(1-2 \varepsilon) \sqrt{2 \log \log \left(a_{m}-a_{m / 2}\right)}$. Now

$$
\sqrt{2 \log \log \left(a_{m}-a_{m / 2}\right)} \approx \sqrt{2 \log \log N^{m}}
$$


for sufficiently large $N$. Therefore

$$
\begin{aligned}
\sum_{m=1}^{\infty} \frac{1}{g\left(n_{m}\right)} \exp \left(-\frac{1}{2} g^{2}\left(n_{m}\right)\right) & \geq c \sum_{m=1}^{\infty} \exp \left(-\frac{1}{2}(1-2 \varepsilon) 2 \log \log N^{m}\right) \\
& \geq c \sum_{m=1}^{\infty} m^{-1+\varepsilon}=\infty
\end{aligned}
$$

Hence by stationary and Lemma 2.1, we obtain

$$
\sum_{m=1}^{\infty} P\left(B_{m}\right)=\infty
$$

Let

$$
B_{m}^{\prime}=\left\{\frac{S\left(a_{m}\right)-S\left(a_{m / 2}\right)}{\sigma\left(a_{m}-a_{m / 2}\right)}>(1-3 \varepsilon) \sqrt{2 \log \log \left(a_{m}-a_{m / 2}\right)}\right\}
$$

and

$$
U_{m}=\frac{S\left(a_{m}\right)-S\left(a_{m / 2}\right)}{\sigma\left(a_{m}-a_{m / 2}\right)}
$$

We will show that

$$
P\left(B_{m}^{\prime}, \text { i.o. }\right)=1
$$

Choose a differential function $f(x)$ such that $\left|f^{\prime}(x)\right| \leq \gamma$ for some $0<\gamma<\infty$ and

$$
\begin{aligned}
0 & \leq I\left\{x>(1-2 \varepsilon) \sqrt{2 \log \log \left(a_{m}-a_{m / 2}\right)}\right\} \\
& \leq f(x) \leq I\left\{x>(1-3 \varepsilon) \sqrt{2 \log \log \left(a_{m}-a_{m / 2}\right)}\right\} \leq 1
\end{aligned}
$$

for any real number $x$. In order to prove $(2.7)$, it is enough to show that

$$
\sum_{m=1}^{\infty} f\left(U_{m}\right)=\infty \quad \text { a.s. }
$$

From (2.6) and (2.8), we get

$$
\sum_{m=1}^{\infty} \operatorname{Ef}\left(U_{m}\right) \geq \sum_{m=1}^{\infty} P\left(B_{m}\right)=\infty
$$


By Markov inequality, we have

$$
\begin{aligned}
& P\left\{\sum_{m=1}^{\infty} f\left(U_{m}\right)<\frac{1}{2} \sum_{m=1}^{n} E f\left(U_{m}\right)\right\} \\
\leq & P\left\{\left|\sum_{m=1}^{n} f\left(U_{m}\right)-\sum_{m=1}^{n} E f\left(U_{m}\right)\right|>\frac{1}{2} \sum_{m=1}^{n} E f\left(U_{m}\right)\right\} \\
\leq & 4 \operatorname{Var}\left(\sum_{m=1}^{n} f\left(U_{m}\right)\right) /\left(\sum_{m=1}^{n} E f\left(U_{m}\right)\right)^{2} \\
\leq & \frac{4}{\sum_{m=1}^{n} E f\left(U_{m}\right)}+\frac{8 \sum_{m=1}^{\infty} \sum_{j=m+1}^{\infty}\left|\operatorname{Cov}\left(f\left(U_{m}\right), f\left(U_{j}\right)\right)\right|}{\left(\sum_{m=1}^{n} \operatorname{Ef}\left(U_{m}\right)\right)^{2}} .
\end{aligned}
$$

Noting that $U_{m}$ and $U_{j}$ are LPQD from the definition of LPQD, and using (ii), $\left(P_{1}\right),\left(P_{2}\right)$ and the regularity of $\sigma(\cdot)$, it follows that, for large number $N$,

$$
\begin{aligned}
& \sum_{m=1}^{\infty} \sum_{j=m+1}^{\infty}\left|\operatorname{Cov}\left(f\left(U_{m}\right), f\left(U_{j}\right)\right)\right| \\
\leq & \sum_{m=1}^{\infty} \sum_{j=m+1}^{\infty} \int_{-\infty}^{\infty} \int_{-\infty}^{\infty}\left|f^{\prime}(x)\right|\left|f^{\prime}(y)\right|\left(P\left\{U_{m} \geq x, U_{j} \geq y\right\}\right. \\
\leq & \left.-P\left\{U_{m} \geq x\right\} P\left\{U_{j} \geq y\right\}\right) d x d y \\
\leq & \sum_{m=1}^{2} \sum_{j=m+1}^{\infty} \operatorname{Cov}\left(U_{m}, U_{j}\right) \quad \sum_{m=1}^{\infty} \operatorname{Cov}\left(\frac{S\left(a_{m}\right)-S\left(a_{m / 2}\right)}{\sigma\left(a_{m}-a_{m / 2}\right)}, \sum_{j=m+1}^{\infty} \frac{S\left(a_{j}\right)-S\left(a_{j / 2}\right)}{\sigma\left(a_{j}-a_{j / 2}\right)}\right) \\
\leq & c \sum_{m=1}^{\infty} \frac{1}{\sigma^{2}\left(a_{m}-a_{m / 2}\right)} \operatorname{Cov}\left(S\left(a_{m}\right)-S\left(a_{m / 2}\right), \sum_{j=m+1}^{\infty}\left\{S\left(a_{j}\right)-S\left(a_{j / 2}\right)\right\}\right) \\
\leq & c \sum_{m=1}^{\infty} \frac{a_{m}-a_{m / 2}}{\sigma^{2}\left(a_{m}-a_{m / 2}\right)} u\left(N^{(m+1) / 2}-N^{m / 2}\right) \\
\leq & c \sum_{m=1}^{\infty}\left(N^{m}\right)^{1-2 \alpha} N^{-\lambda(m+1) / 2} \leq c \sum_{m=1}^{\infty}\left(N^{2 \alpha-1+\lambda / 2}\right)^{-m}<\infty .
\end{aligned}
$$

Combining (2.10)-(2.12) and letting $n \rightarrow \infty$ yields

$$
P\left\{\sum_{m=1}^{\infty} f\left(U_{m}\right)<\infty\right\}=0 .
$$


This proves (2.9) and consequently (2.7). Let

$$
C_{m}=\left\{\frac{S\left(a_{m / 2}\right)}{\sigma\left(a_{m / 2}\right)} \geq-2 \sqrt{2 \log \log a_{m / 2}}\right\} .
$$

It follows from (1.5) and (2.7) that

$$
P\left(B_{m}^{\prime} \cap C_{m}, \text { i.o. }\right)=1 .
$$

It is easy to see that, for $N$ large enough,

$$
\begin{aligned}
& P\left\{\frac{S\left(a_{m}\right)}{\sigma\left(a_{m}\right)}>(1-4 \varepsilon) \sqrt{2 \log \log a_{m}}, \text { i.o. }\right\} \\
\geq & P\left\{Z_{k}>(1-3 \varepsilon) \sqrt{2 \log \log \left(a_{m}-a_{m / 2}\right)}-2 \sqrt{2 \log \log a_{m / 2}}, \text { i.o. }\right\} \\
\geq & P\left\{B_{m}^{\prime} \cap C_{m}, \text { i.o. }\right\}=1 .
\end{aligned}
$$

This implies (2.5).

\section{References}

[1] Y. K. Choi, Limsup results and a uniform LIL for partial sums of an LNQD sequence, Appl. Math. Lett. 24 (2011), no. 2, 138-144.

[2] Y. K. Choi and M. Csörgö, Limsup results and LIL for partial sum processes of a Gaussian random field, Acta Math. Sinica. 24 (2008), no. 9, 1497-1506.

[3] Y. K. Choi, K. S. Hwang, T. S. Kim, Z. Y. Lin, and W. S. Wang, Asymptotic behaviors for partial sum processes of a Gaussian sequence, Acta Math. Hungar. 103 (2004), no. $1-2,43-54$.

[4] J. Esary, F. Proschan, and D. Walkup, Association of random variables with applications, Ann. Math. Statist. 38 (1967), no. 4, 1466-1474.

[5] E. L. Lehmann, Some concepts of dependence, Ann. Math. Statist. 37 (1966), no. 4, 1137-1153.

[6] Y. X. Li and J. F. Wang, The law of the iterated logarithm for positively dependent random variables, J. Math. Anal. Appl. 339 (2008), no. 1, 259-265.

[7] C. M. Newman, Asymptotic independence and limit theorems for positively and negatively dependent random variables, Inequalities in statistics and probability (Lincoln, Neb., 1982), 127-140, IMS Lecture Notes Monogr. Ser., 5, Inst. Math. Statist., Hayward, CA, 1984.

[8] Y. Yang and Y. B. Wang, The asymptotical normality of the renewal process generated by strictly stationary LPQD sequences, Chinese J. Appl. Probab. Statist. 24 (2008), no. 1, 37-42.

Yong-KaB CHOI

Department of Mathematics and RINS

College of Natural Science

Gyeongsang National University

JiNJU 660-701, KOREA

E-mail address: mathykc@naver.com 
KYO-SHIN HWANG

Department of Mathematics

College of Natural Science

Gyeongsang National University

Jinju 660-701, KoreA

E-mail address: hwang0412@naver.com

HeE-Jin MoON

Department of Mathematics

College of Natural Science

Gyeongsang National University

Jinju 660-701, KoreA

E-mail address: mhj0307@hanmail.net 